\title{
Diastolic blood pressure and weight gain predicted future risk for hypertension
}

Bakx JC, van den Hoogen HJ, van den Bosch WJ, et al. Development of blood pressure and the incidence of hypertension in
men and women over an 18-year period: results of the Nijmegen Cohort Study. J Clin Epidemiol 1999 Jun;52:531-8.

QUESTION: Do diastolic blood pressure (BP) and other risk factors predict the development of hypertension in people who are not receiving hypertension treatment at baseline?

Design

Prospective cohort study of men and women in the Nijmegen Cohort Study with 18-year follow-up.

\section{Setting}

6 general practices in the Netherlands.

\section{Participants}

2238 men and women (mean age 35 y, 52\% men, 100\% white) who were not receiving hypertension treatment at baseline.

\section{Assessment of risk factors}

$\mathrm{BP}$, age, familial cardiovascular risk factors, smoking status, physical activity, weight, body mass index (BMI), serum cholesterol level, socioeconomic status, and history of diabetes were measured at baseline. BP, familial risk factors, smoking status, weight, and serum cholesterol level were measured after 18 years of followup. Outcome assessors at follow-up were blinded to the baseline data. Foundation.

For correspondence: J C Bakx, Department of General Practice, University of Nijmegen, 229 HSV P.O. Box $9101,6500 \mathrm{HB}$ Nijmegen, the Netherlands. FAX $+31-24-3541862$

A modified version of the abstract and commentary also appears in Evidence-Based

Nursing

\section{Main outcome measures}

Hypertension was defined in 2 ways: current hyper-

\section{Main results}

Regression analysis in women showed that the risk for developing hypertension was associated with baseline diastolic BP $(\mathrm{p}<0.005)$, weight gain $(\mathrm{p}<0.005)$, and baseline BMI $(p<0.05)$. In men, the risk for developing hypertension was associated with baseline diastolic BP $(\mathrm{p}<0.005)$ and weight gain $(\mathrm{p}<0.005)$ and was inversely tension treatment or diastolic $\mathrm{BP} \geqslant 95 \mathrm{~mm} \mathrm{Hg}$. associated with socioeconomic status $(\mathrm{p}<0.05)$. The table shows the risks for developing hypertension as determined by baseline diastolic BP and weight gain. Women had greater increases in hypertension risk with increasing baseline diastolic $\mathrm{BP}$ and weight gain than did men.

\section{Conclusion}

Diastolic blood pressure and weight gain predicted the 18 -year risk for hypertension in men and women who were not receiving hypertension treatment at baseline.

\section{COMMENTARY}

Several methodological issues in the prospective cohort study by Bakx and colleagues should be noted. The initial evaluation used $2 \mathrm{BP}$ measurements during only 1 visit and used 2 different cuff sizes. For the final screening, however, 1 cuff size was used despite evidence that this variation could result in a systematic difference. At least $12 \%$ of the initial population had hypertension by the diastolic BP criteria but remained in the study because they were not being treated. Furthermore, patients who died between the screenings were not discussed. Despite these issues, however, the conclusions reinforce what has been found in other studies $^{12}$ : Weight gain and baseline BP are predictive of the development of hypertension, especially in women.

What is the clinical utility of this information? The authors suggest that BP measurements should be done regularly in patients with diastolic $\mathrm{BP}>80 \mathrm{~mm} \mathrm{Hg}$ who gain weight. Alternatively, for those with diastolic BP $<75 \mathrm{~mm}$ $\mathrm{Hg}$, the authors maintain that measuring BP every 2 years may be unnecessarily time consuming. The first recommendation is reinforced by other longitudinal studies, but the second cannot be supported by these data because of the methodological issues mentioned above. Given the low cost and non-invasive nature of BP measurement, screening for hypertension should still be recommended every other year for the early detection of this important risk factor.

Richard A Davidson, MD, MPH University of Florida College of Medicine Gainesville, Florida, USA

1 Huang Z, Willett WC, Manson JE, et al. Ann Intern Med 1998; 128:81-8

2 Leitschuh M, Cupples LA, Kannel W, et al. Hypertension 1991;17:22-7.

${ }^{*}$ The 18-year absolute risk for developing hypertension in this group was $7 \%$. †Not significant. 